\title{
The autonomic nervous system in functional bowel disorders
}

\author{
G Tougas
}

\section{Summary}

Communications along the brain-gut axis involve neural pathways as well as immune and endocrine mechanisms. The two branches of the autonomic nervous system are integrated anatomically and functionally with visceral sensory pathways, and are responsible for the homeostatic regulation of gut function. The autonomic nervous system is also a major mediator of the visceral response to central influences such as psychological stress and other central factors.

As presently defined, functional disorders represent a constellation of symptoms, some of which suggest the presence of altered perception while other symptoms point to disordered gastrointestinal function as the cause of the symptoms. There have been a growing number of reports demonstrating disordered autonomic function in subgroups of functional bowel patients. ${ }^{1-3}$ While a number of different methods were used to assess autonomic function, they generally point to decreased vagal (parasympathetic) outflow or increased sympathetic activity in conditions usually associated with slow or decreased gastrointestinal motility. Other studies found either increased cholinergic activity or decreased sympathetic activity in patients with symptoms compatible

Dr G. Tougas.

tougasg@fhs.mcmaster.ca

1200 Main St West

Hamilton, Ontar Ganada with increased motor activity. ${ }^{3}{ }^{4}$ Under certain conditions, altered autonomic balance (including low vagal tone and increased sympathetic activity) may also alter visceral perception.

Autonomic dysfunction could also represent the physiological pathway accounting for many of the extraintestinal symptoms seen in irritable bowel syndrome (IBS) patients, and for some of the frequent gastrointestinal complaints reported by patients with disorders such as chronic fatigue and fibromyalgia.

\section{Introduction}

In recent years, we have undergone a substantial shift in our conceptual definitions of what constitutes functional bowel disorders and IBS. In general, we now view these conditions as a group of disorders or clinical entities characterised by the presence of, to a varying degree, chronic pain, discomfort, and disordered gastrointestinal function. While this type of clinical definition corresponds to how these patients present, it has proved difficult to apply in the search for the pathophysiological factor(s) potentially involved in these conditions. While much remains to be done in that regard, it is increasingly accepted that these conditions are multifactorial, and that the symptoms experienced by two individual patients, although somewhat similar, may in fact result from a number of different aetiologies.

Abnormalities of motor and secretory function as well as altered enteric neural function can be identified in many patients with functional disorders (fig 1). However, most patients have no demonstrable motor dysfunction and the abnormalities that have been identified vary from study to study, each of these abnormalities being present in small and very specific groups of patients which do not represent the majority of those seen in clinical practice. The concept of these conditions being primarily motility or enteric nervous disorders is now also generally viewed as failing to account for many of the other characteristics that are present in these patients, in particular the discomfort and pain often reported in the presence of apparently normal gastrointestinal function.

In many patients with functional bowel disorders, the presence of altered visceral perception to various visceral stimuli, such as distension, has been identified. This issue of altered visceral perception in IBS is extensively discussed elsewhere. It remains important to point out that while a primary disorder of visceral sensory perception provides a plausible explanation for many of the symptoms reported in functional bowel disorders, it remains a largely unproved

Abbreviations used in this paper: IBS, irritable bowel syndrome.
Figure 1 Putative pathogenesis of dyspepsia. ANS, autonomic nervous system; $H P$, Helicobacter pylori.

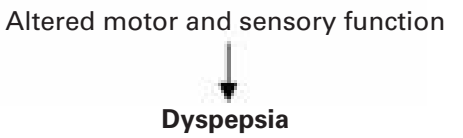

afferent

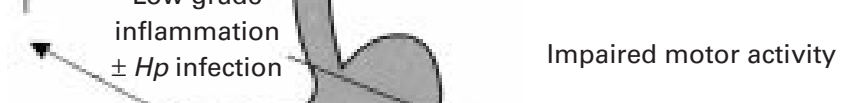

Dyspepsia 
concept at the clinical level. In specific situations such as the development of chronic symptoms following an acute inflammatory or infectious event (such as post-infectious irritable bowel syndrome), altered function of the normal sensory mechanisms, either within the gut wall or along visceral afferent pathways, are conceivably possible, at least in the short term. It is more difficult at this point to implicate altered visceral sensory pathways in the pathogenesis of functional symptoms such as diarrhoea or bloating. Such symptoms are present in many postinfectious IBS patients, but also in patients where there is no history of any acute inflammatory or infectious precipitant prior to the development of symptoms. Similarly, the hypothesis of altered visceral sensory perception as the only factor involved in functional disorders fails to account for the very high association of stress, psychologically traumatic experiences, and emotional distress with the development and persistence of functional bowel symptoms. Unless one extends the concept of altered visceral sensory perception to include the cortical centres associated with visceral perception, or a central nervous system modulation of the afferent and spinal pathways involved with perception of visceral stimuli, it is somewhat difficult to build a conceptual framework capable of reconciling cortical events such as emotions with the peripheral sensory pathways involved with visceral sensory function. If a pragmatic approach is used to reconcile the various components identified in the clinical expression of functional bowel disorders with an overall concept of the pathophysiological factors involved, several conditions must be met. Firstly, the condition (IBS) involves central as well as peripheral sites: pain is ultimately a cortical event while events such as diarrhoea and mucus production imply that there is also dysfunction of the gut per se. Therefore, functional disorders must to some extent involve the brain as well as the gut. Secondly, if visceral factors such as an infectious event in the gut affect the cortical response to visceral stimuli, and if conversely, psychological events can alter the function of the gut, gut-brain and brain-gut communication must be one of the important modulators involved. Therefore, it seems logical that the autonomic nervous system, which is the primary pathway involved in brain-gut communication, plays an important role in functional bowel disorders (fig 2).

The autonomic nervous system could be simply a conduit through which the central nervous system controls visceral function or it may be that primary alterations in visceral autonomic function are one of the pathophysiological factors involved in these conditions. In the following sections we will examine the possible role of the autonomic nervous system in functional disorders and some of the therapeutic opportunities that modulation of autonomic function may provide in the management of functional disorders.

Functional bowel disorders: brain-gut, gut-brain, or both?

In assessing the role of the autonomic nervous system in conditions such as functional bowel disorders, it is essential to remember the homeostatic nature of autonomic function. While many local gastrointestinal stimuli will elicit responses and reflexes whose involvement is essentially limited to the immediate region that was primarily stimulated, when more intense or potentially noxious stimuli occur, other systems, including the central nervous system, are also involved in addition to the gastrointestinal tract. Transmission of information to the central nervous system allows for elaboration of an integrated homeostatic response that may include a behavioural as well as a physiological response. In this type of response, both visceral and autonomic nervous pathways are essential.

Many of the systemic responses elicited by visceral stimuli are produced through autonomic reflexes. These reflexes, which occur primarily through the brain stem, may not be associated with conscious perception of the sensory stimulus. However, other visceral sensory stimuli will be sufficiently intense as to be felt and to elicit pain or discomfort.

The type and degree of the autonomic reflex response to a specified visceral stimulus depends on the location, type, and intensity of the stimulus. If prior sensitisation of the visceral afferent pathways has resulted in a state of hyperalgesia or allodynia, the reflex response may be exaggerated, while a peripheral sensory neuropathy may be associated with a decreased reflex response. Similarly, psychological factors such as increased vigilance, anxiety, and depression are also likely to affect autonomic reflexes. ${ }^{5}$

\section{Assessing autonomic function}

A number of methods have been developed to assess specific aspects of autonomic nervous function. Some of the older approaches, which were very cumbersome and involved rather complex measurements, have been largely abandoned. In recent years, the development of techniques based on the autonomic modulation of heart rate function have largely replaced other methods because of their simplicity and validity as markers of vagal as well as sympathetic function. ${ }^{6}$ The use of techniques such as power spectral analysis of heart rate variability now provide a simple and accurate measure of the respective outflow of the vagal and sympathetic branches of the autonomic nervous system. ${ }^{4}$ As

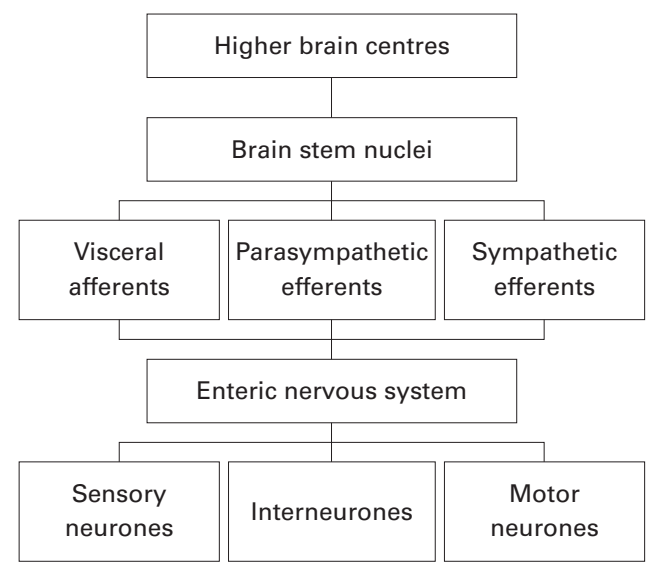

Figure 2 Autonomic nervous system alternate view. 


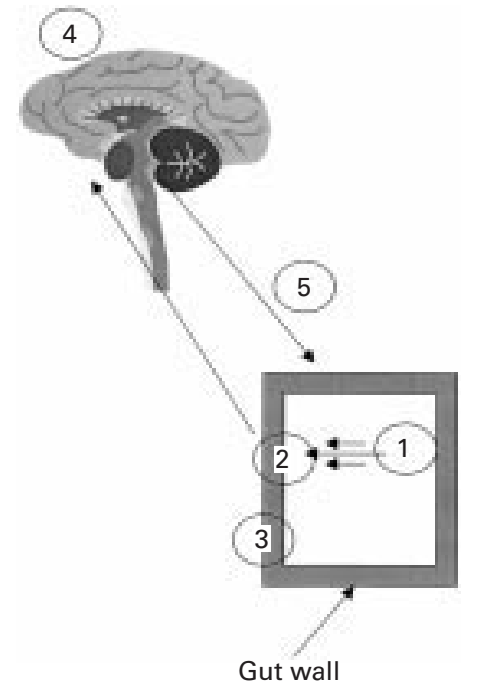

1. Abnormal stimulation of normal GI receptors

2. Abnormal response of $\mathrm{GI}$
receptors to normal stimuli

3. Abnormal intrinsic GI motility

4. Abnormal central perception of normal $\mathrm{Gl}$ afferent signals

5. Abnormal central stimulation of GI secretion/motility via efferent pathways

Figure 3 Functional bowel disease. with non-cardiac chest pain, we have found the opposite; those patients with increased visceral sensitivity to oesophageal acid infusion had higher resting sympathetic tone and decreased vagal activity, suggesting that the abnormalities may differ according to the gut region involved (foregut $v$ hindgut). ${ }^{1}$

However, it is difficult from these associations to ascribe a definite causative role to any type of autonomic dysfunction in functional disorders until studies aimed at restoring a more normal basal autonomic balance are also shown to change the altered visceral perception and function that is present in these patients. Central and emotional factors, which are well known to be associated with functional gut symptoms, are also capable of altering autonomic balance. It would be naïve not to consider that in a large number of cases where central as well as autonomic factors are involved it is the effects of the former that may lead to the occurrence of the latter and of gastrointestinal symptoms (fig 3).

the method requires only a surface electrocardiogram, it can be done in almost any setting. ${ }^{6}$

Autonomic function and bowel disorders In recent years, there have been a number of reports suggesting that functional bowel disorders are associated with autonomic disturbance. ${ }^{7}$ However, none of these studies established more than an association, and should not at this point be interpreted to indicate that altered autonomic function is causally related to functional disorders.

In animals, surgical ablation of coeliac or mesenteric ganglia has profound effects on gastrointestinal function. ${ }^{8}$ Furthermore, tumour invasion of the sympathetic ganglia can result in intestinal and/or colonic pseudoobstruction in patients with metastatic cancer. ${ }^{9}$ Conditions affecting autonomic function, such as Parkinsonism and autonomic neuropathies, also have profound effects on gut function, as do the degenerative neuropathies associated with diabetes and amyloidosis. ${ }^{10}$ However, these alterations are clearly not the direct cause of symptoms in the majority of patients with functional symptoms.

Altered autonomic function may also be involved in other gastrointestinal conditions, such as gastro-oesophageal reflux disease and neuropathic upper gastrointestinal motility disorders. ${ }^{11}{ }^{12}$ More interestingly, such vagal abnormalities have also been identified in patients with functional gut disorders, including functional dyspepsia and colonic inertia. ${ }^{13} 14$

More recently, Aggarwal et al have shown convincingly that a subgroup of patients with IBS also showed various autonomic abnormalities. ${ }^{3}$ However, the findings were varied and not present in all patients in their cohort. Patients with increased sympathetic activity and low vagal tone tended to have constipation, whereas diarrhoea prone patients primarily showed increased parasympathetic activity. Another group has shown that functional abdominal pain without any motility abnormalities was associated with increased basal parasympathetic activity and lower sympathetic activity. ${ }^{2}$ In patients

\section{Conclusions}

There is increasing agreement that autonomic abnormalities can often be associated with functional disorders of the gut. We still have to gain a better understanding of the mechanisms responsible for the symptoms and of their fate with restoration of autonomic function. Until then the exact role of altered autonomic function in the pathogenesis of functional disorders will remain as poorly defined as that of the other putative mechanisms involved in these disorders. Once more, the urgent need for studies focusing on the mechanisms of disease rather than its clinical manifestation, and on pathophysiology rather than symptomatology, is apparent.

1 Spaziani RM, Djuric V, Kamath MV, et al. A low resting vagal tone predicts response to acid perfusion in patien with esophageal symptoms. Gastroenterology 1996;110:A762.

2 Jorgensen LS, Christiansen P, Raundahl U, et al. Autonomic nervous system function in patients with functional abdominal pain. Scand F Gastroenterol 1993;28:63-8.

3 Aggarwal A, Cutts TF, Abell TL, et al. Predominant symptoms in irritable bowel syndrome correlate with specific autonomic nervous system abnormalities. Gastroenterology 1994;106:945-50.

4 Kamath MV, Fallen EL. Power spectral analysis of heart rate variability: a non-invasive signature of cardiac autonomic function. Crit Rev Biomed Eng 1993;21:245-311.

5 Almy TP. The irritable bowel syndrome. Back to square one. Dig Dis Sci 1980;25:401-3.

6 Tougas G, Kamath M, Watteel G, et al. Modulation of neuClin Sci 1997;92:167-74

7 Bharucha AE, Camilleri M, Low PA, et al. Autonomic dysfunction in gastrointestinal motility disorder. Gut 1993;34 397-401.

8 Popielski L. Zur physiologie des plexus coeliacus (experimentelle untersuchung). Arch Anat Physiol 1903:338-60.

Ogilvie $\mathrm{H}$. Large intestine colic due to sympathetic invasion. BMF 1948;2:671-3.

10 Camilleri M. Disorders of gastrointestinal motility in neurologic diseases. Mayo Clin Proc 1990;65:825-6.

11 Chakraborty TK, Ogilvie AL, Heading RC, et al. Abnormal cardiovascular reflexes in patients with gastro-oesophageal cardiovascular reflexes in

12 Ogilive AL, James PD, Atkinson M. Impairment of vagal function in reflux oesophagitis. $Q \mathcal{F}$ Med 1985;54;61-74.

13 Haug TT, Svebak S, Hausken T, et al. Low vagal activity as mediating mechanism for the relationship between personality factors and gastric symptoms in functional dyspepsia. Psychosom Med 1994;56:181-6.

4 Latimer P, Sarna S, Campbell D, et al. Colonic motor and myoelectrical activity: a comparative study of normal subjects, psychoneurotic patients and patients with irritable bowel syndrome. Gastroenterology 1981;80:893-900. rocardiac function by oesophageal stimulation in humans. 